\title{
Incoherent $\rho^{0}$ electroproduction off nuclei
}

\author{
T. Falter*, K. Gallmeister and U. Mosel \\ Institut fuer Theoretische Physik, Universitaet Giessen \\ D-35392 Giessen, Germany
}

(December 30, 2002)

\begin{abstract}
In the present paper we investigate incoherent $\rho^{0}$ electroproduction off complex nuclei. We derive a novel, simple expression for the incoherent electroproduction cross section in which one can clearly separate the final state interactions of the reaction products from the 'initial state interactions' of the photon that give rise to nuclear shadowing. In the special case of purely absorptive final state interactions we deduce from our expression the known Glauber result. A more realistic treatment of the final state interactions within a transport model is then used to compare our predictions with experimental data from the HERMES experiment.
\end{abstract}

PACS numbers: 25.20.Lj, 24.10.Eq, 24.10.Nz, 25.75.Dw

*email: Thomas.Falter@theo.physik.uni-giessen.de 


\section{INTRODUCTION}

High energy meson electroproduction off complex nuclei offers a promising tool to study the physics of hadron formation. The nuclear environment makes it possible to investigate the properties of hadrons immediately after their creation. In addition one can vary the energy and virtuality of the exchanged photon to examine the phenomenon of color transparency [1].

In the present paper we study exclusive incoherent $\rho^{0}$ electroproduction off ${ }^{14} \mathrm{~N}$ and ${ }^{84} \mathrm{Kr}$ in the energy regime of the HERMES experiment $[2,3]$. In this kinematic regime the coherence length of the photon's $\rho^{0}$ component is large and quantum mechanical interference effects play an important role. In [4] we presented a method to account for this so-called shadowing effect in photoproduction within a semi-classical BUU transport model which we now apply for electron induced reactions. We distinguish between the 'initial state interactions' of the photon that give rise to shadowing and the final state interactions (FSI) of the produced particles. The coupled channel treatment of the FSI within the transport model allows for a broader spectrum of FSI than the usually used Glauber theory [5]. In addition we account for Fermi motion and Pauli blocking during the reaction and take the finite lifetime of unstable reaction products into account.

The time that the products of an elementary photon-nucleon interaction need to evolve to physical hadrons is called formation time. Any change in the reaction probability of a hadron $h$ during its formation time affects the so-called nuclear transparency

$$
T_{A}=\frac{\sigma_{\gamma^{*} A \rightarrow h X}}{A \sigma_{\gamma^{*} N \rightarrow h X}}
$$

if the formation length, i.e. the length that $h$ travels during its formation time, exceeds the average internucleon distance $(\approx 1.7 \mathrm{fm})$ inside the nucleus.

In the case of diffractive vector meson electroproduction $\left(\gamma^{*} N \rightarrow V N\right)$ the virtual photon initially produces a colorless $q \bar{q}$-pair whose size is expected to decrease with increasing photon virtuality $Q^{2}[6]$. As long as the $q \bar{q}$-pair is very small, i.e. in the early stage of its evolution into the physical vector meson, it mainly reacts via its color dipole moment. This leads to a cross section that is quadratic in the $q \bar{q}$-pair's size. At large enough energies the $q \bar{q}$-pair is frozen in this small sized configuration over a distance that, because of time dilatation, can exceed the diameter of a nucleus. This effect leads to a large nuclear transparency for vector meson production at large photon energy $\nu$ and virtuality $Q^{2}$ (color transparency). For a detailed review of color transparency see e.g. [7] and references therein.

It is not clear whether the energy and the virtuality of the photon in the HERMES experiment is large enough to see an onset of color transparency. In the present paper we do not find evidence for a finite formation time of the produced vector meson. The ${ }^{14} \mathrm{~N}$ data for the transparency ratio as function of the coherence length is compatible with the assumption that the diffractively produced $\rho^{0}$ starts interacting with a hadronic cross section right after its production. Kopeliovich et al. [6] point out that this might be an accidental consequence of the specific correlation between the $Q^{2}$ of the photon and the coherence length of the $\rho^{0}$ in the HERMES data. In their work a light-cone QCD formalism was used to incorporate formation and coherence length effects in coherent and incoherent vector meson electroproduction. In addition the effect of gluon shadowing was studied, but 
found to be negligible in the case of incoherent $\rho$ production. In Ref. [6] the decrease of the transparency ratio due to the finiteness of the $\rho^{0}$ lifetime was discussed as well. This effect is automatically included within our transport model. Our coupled channel calculation shows in addition that the effects of side-feeding in the FSI are unimportant because of the kinematic cuts of the HERMES experiment. Therefore Glauber theory seems to be appropriate for these reactions. However, we find that for heavy nuclei the kinematic cuts lead to an additional reduction of the transparency ratio due to elastic FSI which scatter the produced particle out of the acceptance window. One must therefore be careful which cross section one uses to describe the FSI in Glauber theory.

Our paper is structured in the following way: In Section II A we show how we describe the electron-nucleon interaction and how we account for shadowing within our model. In the limit of purely absorptive FSI our result for the incoherent vector meson production cross section turns out to be equivalent to the known Glauber result of Huefner et al. [8]. However, in contrast to the result of Ref. [8] our expression allows for an intuitive physical interpretation. The transport model itself is sketched in Section IIB. The results of our calculations for the exclusive incoherent $\rho^{0}$ production cross section within the coupled channel treatment are presented in Section III in comparison with experimental data from the HERMES experiment. We close with a short summary and outlook in Section IV.

\section{MODEL}

\section{A. Shadowing}

In the one photon exchange picture the interaction of an electron and a nucleon (or nucleus) can be reduced to the reaction of a virtual photon on the hadronic target. The exchange of more than one photon is suppressed by further factors of the fine structure constant $\alpha_{e m}$. We follow the method of Friberg and Sjostrand [9] and use the event generator PYTHIA v6.2 [10] to describe the interaction of the (virtual) photon and a nucleon. The basic idea is that in a photon hadron collision the photon not necessarily interacts as a point particle (direct interaction) but might fluctuate into a vector meson $V=\rho^{0}, \omega, \phi, J / \Psi$ (vector meson dominance) or perturbatively branch into a $q \bar{q}$ pair before the interaction (generalized vector meson dominance, GVMD). Within the PYTHIA model it turns out that the latter is very unlikely in the kinematic regime of the HERMES experiment (photon energy $\nu \approx 10-20 \mathrm{GeV}, Q^{2} \approx 0.5-5 \mathrm{GeV}^{2}$ ) as can be seen from Fig. 1 where we show the contribution of the different photon components to the total $\gamma^{*} N$ cross section at an invariant mass $W=5 \mathrm{GeV}$. In most cases the photon will therefore scatter deep inelastically from a parton of the target nucleon or fluctuate into a vector meson before it reaches the nucleon. In the latter case the vector meson might either scatter diffractively from the nucleon or a hard scattering between the constituents of the vector meson and the nucleon might take place. The hard scattering leads to the excitation of one or two hadronic strings which finally fragment into hadrons. We assume that the hadrons that emerge from the edges of these strings, i.e. those that contain quarks not originating from the string fragmentation, can interact with a hadronic cross section right after the photon nucleon reaction. Consequently, 
for the diffractively produced vector meson we do not use a formation time since none of its constituents arise from a string fragmentation.

If the struck nucleon is embedded in a nucleus one has to account for its Fermi motion and binding energy as well as Pauli blocking of final state nucleons. In addition one has to be aware that the nuclear environment influences the VMD part of the photon nucleon interaction, since the vector meson components get modified on their way through the nuclear medium to the interaction point.

We express the physical photon state $|\gamma\rangle$ in terms of vector meson states $|V\rangle$ and a state $\left|\gamma_{0}\right\rangle$ which consists of the point-like photon and the GVMD part. This makes sense if one assumes that the GVMD part does not get shadowed or as in our case is unimportant for kinematic reasons:

$$
|\gamma\rangle=\left(1-\sum_{V=\rho, \omega, \phi, J / \Psi} \frac{e^{2}}{2 g_{V}^{2}} F_{V}^{2}\right)\left|\gamma_{0}\right\rangle+\sum_{V=\rho, \omega, \phi, J / \Psi} \frac{e}{g_{V}} F_{V}|V\rangle .
$$

The formfactor

$$
F_{V}^{2}=\left(\frac{W^{2}}{Q^{2}+W^{2}}\right)^{3}\left[1+0.5 \frac{4 m_{V}^{2} Q^{2}}{\left(m_{V}^{2}+Q^{2}\right)^{2}}\right]\left(\frac{m_{V}^{2}}{m_{V}^{2}+Q^{2}}\right)^{2}
$$

is taken from [9] and also accounts for contributions of longitudinal photons. In Eq. (2.2) $\nu$ denotes the energy of the photon, $Q^{2}$ its virtuality and $m_{V}$ the mass of the vector meson $V$. $W$ is the invariant mass of the photon nucleon system.

For a photon with momentum $k \vec{e}_{z}$ the wave function of the component $V$ can be written as

$$
\psi_{V}^{(0)}(\vec{b}, z)=\chi(\vec{b}) e^{i k z}
$$

On the way through the nucleus the vector meson components get modified. Consider an ensemble of $A$ nucleons at positions $\left\{\vec{r}_{i}\right\}=\left\{\left(\vec{s}_{i}, z_{i}\right)\right\}$ which are labeled in such a way that $z_{1}<z_{2}<\ldots<z_{A}$. According to Glauber theory [11] the wave function $\psi_{V}$ behind the first nucleon looks like

$$
\begin{aligned}
\psi_{V}^{(1)}(\vec{b}, z) & =\psi_{V}^{(0)}(\vec{b}, z)-\Gamma_{V}\left(\vec{b}-\vec{s}_{1}\right) \psi_{V}^{(0)}\left(\vec{b}, z_{1}\right) e^{i k_{V}\left(z-z_{1}\right)} \\
& =\chi(\vec{b})\left(e^{i k z}-\Gamma_{V}\left(\vec{b}-\vec{s}_{1}\right) e^{i q_{V} z_{1}} e^{i k_{V} z}\right)
\end{aligned}
$$

if one neglects off-diagonal scattering from one vector meson component into another (diagonal approximation). The phase factor in the second term arises from putting the vector meson component on its mass shell, i.e. $k_{V}=\sqrt{\nu^{2}-m_{V}^{2}}$ and $q_{V}=k-k_{V}$ is the corresponding momentum transfer. The profile function $\Gamma_{V}$ is related to the elastic vector meson nucleon scattering amplitude $f_{V}$ in the following way:

$$
\Gamma_{V}(\vec{b})=\frac{1}{2 \pi i \vec{k}_{V}} \int e^{i \vec{k}_{t} \cdot \vec{b}} f_{V}\left(\vec{k}_{t}\right) d^{2} k_{t}
$$

After passing the second nucleon the wave function takes on the form 


$$
\psi_{V}^{(2)}(\vec{b}, z)=\chi(\vec{b})\left(e^{i k z}-\Gamma_{V}\left(\vec{b}-\vec{s}_{2}\right) e^{i q_{V} z_{2}} e^{i k_{V} z}-\Gamma_{V}\left(\vec{b}-\vec{s}_{1}\right)\left(1-\Gamma_{V}\left(\vec{b}-\vec{s}_{2}\right) e^{i q_{V} z_{1}} e^{i k_{V} z}\right)\right.
$$

At position $z$ the whole ensemble of $A$ nucleons has led to the modification

$$
\begin{aligned}
\psi_{V}^{(A)}(\vec{b}, z) & =\chi(\vec{b})\left(e^{i k z}-\sum_{j=1}^{A} \Gamma_{V}\left(\vec{b}-\vec{s}_{j}\right) e^{i q_{V} z_{j}} \prod_{k \neq j}^{A}\left[1-\Gamma_{V}\left(\vec{b}-\vec{s}_{k}\right) \Theta\left(z_{k}-z_{j}\right)\right] \Theta\left(z-z_{k}\right) e^{i k_{V} z}\right) \\
& =\left\{1-\sum_{j=1}^{A} \Gamma_{V}\left(\vec{b}-\vec{s}_{j}\right) e^{i q_{V}\left(z_{i}-z\right)} \prod_{k \neq j}^{A}\left[1-\Gamma_{V}\left(\vec{b}-\vec{s}_{k}\right) \Theta\left(z_{k}-z_{j}\right)\right] \Theta\left(z-z_{k}\right)\right\} \psi_{V}^{(0)}(\vec{b}, z) \\
& =\left\{1-\Gamma_{V}^{(A)}\left(\vec{b}, z ;\left\{\vec{r}_{i}\right\}\right)\right\} \psi_{V}^{(0)}(\vec{b}, z)
\end{aligned}
$$

Hence, the physical photon state changes on its way through the nucleus to position $\vec{r}=(\vec{b}, z)$ according to

$$
|\gamma(\vec{r})\rangle=\left(1-\sum_{V=\rho, \omega, \phi, J / \Psi} \frac{e^{2}}{2 g_{V}^{2}} F_{V}^{2}\right)\left|\gamma_{0}\right\rangle+\sum_{V=\rho, \omega, \phi, J / \Psi} \frac{e}{g_{V}} F_{V}\left(1-\overline{\Gamma_{V}^{(A)}}(\vec{r})\right)|V\rangle
$$

where the nuclear profile function from (2.7) must be averaged over the positions of the nucleons in the nucleus

$$
\begin{aligned}
\overline{\Gamma_{V}^{(A)}}(\vec{b}, z) & =\left\langle 0\left|\Gamma_{V}^{(A)}\left(\vec{b}, z ;\left\{\vec{r}_{i}\right\}\right)\right| 0\right\rangle \\
& =\int_{-\infty}^{z} d z_{i} n\left(\vec{b}, z_{i}\right) \frac{\sigma_{V N}}{2}\left(1-i \alpha_{V}\right) e^{i q_{V}\left(z_{i}-z\right)} \exp \left[-\frac{1}{2} \sigma_{V N}\left(1-i \alpha_{V}\right) \int_{z_{i}}^{z} d z_{k} n\left(\vec{b}, z_{k}\right)\right] .
\end{aligned}
$$

To get to the second line of Eq. (2.9) we have followed the way of [11], i.e. making use of the independent particle model and the large $A$ limit and used the optical theorem to express the elastic forward scattering amplitude in terms of the total $V N$ cross section $\sigma_{V}$ :

$$
f_{V}(\overrightarrow{0})=\frac{i k_{V}}{4 \pi} \sigma_{V}\left(1-i \alpha_{V}\right)
$$

The nucleon number density is denoted by $n(\vec{r})$ and normalized to $A$.

Neglecting any influence of the FSI, the reaction amplitude for the process $\gamma N \rightarrow f$ on a nucleon at position $\vec{r}$ inside a nucleus changes compared to the vacuum due to these 'initial state interactions' of the photon:

$$
\langle f|\hat{T}| \gamma\rangle \rightarrow\langle f|\hat{T}| \gamma(\vec{r})\rangle
$$

By comparing Eq. (2.1) and (2.8) one sees that to account for shadowing in incoherent photoproduction one only has to multiply the amplitude of each vector meson states $|V\rangle$ by the corresponding factor $\left(1-\overline{\Gamma_{V}^{(A)}}(\vec{r})\right)$. This is equivalent to the method we used in [4]. 
We now compare our method of describing shadowing of incoherent electroproduction with conventional Glauber results. We therefore calculate the incoherent vector meson electroproduction cross section off a nucleus and assume purely absorptive FSI for the moment. After averaging over all positions $\vec{r}=(\vec{b}, z)$ we get:

$$
\begin{aligned}
\sigma_{\gamma A \rightarrow V A^{*}}= & \sigma_{\gamma N \rightarrow V N} \int d^{2} b \int_{-\infty}^{\infty} d z n(\vec{b}, z) \\
\times & \left|1-\int_{-\infty}^{z} d z_{i} n\left(\vec{b}, z_{i}\right) \frac{\sigma_{V}}{2}\left(1-i \alpha_{V}\right) e^{i q_{V}\left(z_{i}-z\right)} \exp \left[-\frac{1}{2} \sigma_{V}\left(1-i \alpha_{V}\right) \int_{z_{i}}^{z} d z_{k} n\left(\vec{b}, z_{k}\right)\right]\right|^{2} \\
& \times \exp \left[-\sigma_{V}^{\text {inel }} \int_{z}^{\infty} d z^{\prime} n\left(\vec{b}, z^{\prime}\right)\right]
\end{aligned}
$$

In (2.12) we again neglect off-diagonal scattering, i.e. $V$ production is triggered by the $V$ component of the photon only. The last factor in (2.12) includes the inelastic $V N$ cross section $\sigma_{V}^{\text {inel }}$ and accounts for the FSI.

The production process described by Eq.(2.12) can be understood as follows: The incoherent vector meson production takes place on a nucleon at position $(\vec{b}, z)$. The production is triggered either directly by the photon or via an intermediate vector meson which was earlier produced on a nucleon at position $\left(\vec{b}, z_{i}\right)$ without excitation of the nucleus. The interactions of this vector meson on its way from $z_{i}$ to position $z$ are of optical potential type and leave the nucleus in its ground state. This propagation is described by the second exponential in (2.12). The interference between the direct and the indirect process leads to shadowing. The nucleus gets excited in the incoherent reaction at position $z$. The possibility that the vector meson is lost on its way from position $z$ out of the nucleus is taken care of by the last exponential.

Expression (2.12) formally differs only slightly from the approximate result for the incoherent vector meson production cross section which is given without derivation in Ref. [11]. The latter can be obtained by making the following replacements in (2.12):

$$
e^{i q_{V}\left(z_{i}-z\right)} \rightarrow e^{i q_{V} z_{i}}, \quad \sigma_{V}^{i n e l} \rightarrow \sigma_{V}
$$

The last replacement simply means that one neglects the possibility of elastic $V N$ scattering whereas the occurrence of the different phase factor is unclear. The Authors of Ref. [8] have given a seemingly different result for incoherent $V$ electroproduction:

$$
\begin{aligned}
\sigma_{\gamma A \rightarrow V A^{*}}=\sigma_{\gamma N} \rightarrow V N & \int d^{2} b\left\{\int_{-\infty}^{+\infty} d z n(\vec{b}, z) e^{-\sigma_{V}^{i n e l} T_{z}(\vec{b})}\right. \\
+ & \frac{1}{2} \frac{\sigma_{V}}{\sigma_{V}^{e l}}\left(\sigma_{V}^{i n e l}-\sigma_{V}^{e l}\right) \int_{-\infty}^{+\infty} d z_{1} n\left(\vec{b}, z_{1}\right) \int_{z_{1}}^{+\infty} d z_{2} n\left(\vec{b}, z_{2}\right) \\
& \times \cos \left[q_{V}\left(z_{1}-z_{2}\right)\right] e^{-\frac{1}{2}\left(\sigma_{V}^{i n e l}-\sigma_{V}^{e l}\right) T_{z_{2}}(\vec{b})-\frac{1}{2} \sigma_{V} T_{z_{1}}(\vec{b})}
\end{aligned}
$$




$$
\left.-\frac{1}{4} \frac{\left(\sigma_{V}\right)^{2}}{\sigma_{V}^{e l}}\left|\int_{-\infty}^{+\infty} d z n(\vec{b}, z) e^{i q_{V} z} e^{-\frac{1}{2} \sigma_{V} T_{z}(\vec{b})}\right|^{2}\right\}
$$

with $T_{z}(\vec{b})=\int_{z}^{\infty} n\left(\vec{b}, z^{\prime}\right) d z^{\prime}$ and $T(\vec{b})=T_{z=-\infty}(\vec{b})$. Expression (2.14) can be interpreted as follows: The last term represents the coherent $V$ photoproduction cross section which is subtracted from the inclusive $V$ photoproduction cross section to yield the incoherent part. In Appendix A we show that the physically more transparent expression (2.12) is mathematically identical to (2.14).

\section{B. Transport model}

Up to now we have ignored the effects of Fermi motion, binding energies and Pauli blocking of final state nucleons in the process described by (2.11). In addition we neglected the finite life time of the $\rho^{0}$ and took only absorptive FSI into account. These shortcomings are avoided when using the model of Ref. $[4,12]$. The propagation of the final state $|f\rangle$ through the nucleus is treated within a semi-classical transport model based on the BoltzmannUehling-Uhlenbeck (BUU) equation. The BUU equation describes the time evolution of the phase space density $f_{i}(\vec{r}, \vec{p}, t)$ of particles of type $i$ that can interact via binary reactions. Besides the nucleons these particles involve baryonic resonances and mesons $(\pi, \eta, \rho, K, \ldots)$ that are produced either in the primary reaction or during the FSI. For a particle species $i$ the BUU equation can be written as:

$$
\left(\frac{\partial}{\partial t}+\frac{\partial H}{\partial \vec{r}} \frac{\partial}{\partial \vec{r}}-\frac{\partial H}{\partial \vec{r}} \frac{\partial}{\partial \vec{p}}\right) f_{i}(\vec{r}, \vec{p}, t)=I_{\text {coll }}\left[f_{1}, \ldots f_{i}, \ldots, f_{M}\right] .
$$

For baryons the Hamilton function $H$ includes a mean field potential which depends on the particle position and momentum. The collision integral on the right hand side accounts for the creation and annihilation of particles of type $i$ in a collision as well as elastic scattering from one position in phase space into another. For fermions Pauli blocking is taken into account in $I_{\text {coll }}$ via blocking factors. The BUU equations of each particle species $i$ are coupled via the mean field and the collision integral. The resulting system of coupled differentialintegral equations is solved via a test particle ansatz for the phase space density. For details of the transport model see Ref. [12].

The classes of FSI that are included in the transport model goes far beyond what can be achieved within Glauber theory. As a result the finally observed $\rho^{0}$ does not need to be created in the primary reaction but might be produced during the FSI via side feeding. It is therefore clear that a purely absorptive treatment of the FSI as in Glauber theory can only be used if one is sure that one has eliminated the possibility of side feeding by applying enough constraints on the observable (see [4] for details).

We also stress that within our model instable particles might decay during their propagation through the nucleus. In case of the $\rho^{0}$ this means that both pions have to escape the nucleus without further rescattering to make the identification of the $\rho^{0}$ still possible. 


\section{RESULTS}

Before we turn to nuclear targets we first verify that the input of our model is reasonable. We therefore look at exclusive $\rho^{0}$ production off hydrogen. We use the same kinematical cuts as in the HERMES experiment [13], i.e. the final state has to consist of two oppositely charged pions with invariant mass between $0.6 \mathrm{GeV}$ and $1 \mathrm{GeV}$. The four momentum transfer $\left|t-t_{\text {max }}\right|$ between the virtual photon and the $\pi^{+} \pi^{-}$-pair has to be smaller than $0.4 \mathrm{GeV}^{2}$ and we apply the exclusivity measure

$$
\Delta E=\frac{p_{Y}^{2}-m_{N}^{2}}{2 m_{N}}<0.4 \mathrm{GeV},
$$

where $m_{N}$ denotes the nucleon mass and

$$
p_{Y}=p_{N}+p_{\gamma}-p_{\rho}
$$

the 4-momentum of the undetected final state. In Eq. (3.2) $p_{\gamma}$ and $p_{\rho}$ denote the 4-momenta of the incoming photon and the detected $\pi^{+} \pi^{-}$pair and $p_{N}$ is the 4-momentum of the struck nucleon which, for the calculation of $p_{Y}$, is assumed to be at rest. In Fig. 2 we compare our calculation of the exclusive $\rho^{0}$ production cross section off hydrogen with experimental data [13-15]. In the whole $Q^{2}$ region covered by the HERMES experiment we find very good agreement for a broad range of the invariant mass $W$ of the photon nucleon system. Also the slope of the differential production cross section is reproduced very well by the PYTHIA model as can be seen from the solid line in Fig. 3 where we show our calculation of $\frac{d \sigma}{d t}\left(\gamma^{*} p \rightarrow \rho^{0} p\right)$ together with the HERMES data [2].

For our calculations on exclusive $\rho^{0}$ electroproduction off nuclei we again use the kinematic cuts of the HERMES collaboration [2,3]. This means that we restrict our exclusivity measure to the region

$$
-2 \mathrm{GeV}<\Delta E<0.6 \mathrm{GeV},
$$

and introduce a lower boundary for the four-momentum transfer $\left|t-t_{\text {max }}\right|>0.09 \mathrm{GeV}^{2}$ as imposed by the HERMES collaboration to get rid of coherently produced $\rho^{0}$. From the dashed line in Fig. 3 one sees that the differential $\rho^{0}$ electroproduction cross section off ${ }^{14} \mathrm{~N}$ is again in excellent agreement with the HERMES data [2]. Throughout our calculations the effect of the nucleon potential turns out to be negligible. This is reasonable since the involved energies are much larger than the typical binding energies which are in the order of a few $\mathrm{MeV}$. A combined effect of Fermi motion and Pauli blocking on the incoherent differential production cross section is visible at $\left|t-t_{\text {max }}\right|<0.1 \mathrm{GeV}^{2}$. At $\left|t-t_{\text {max }}\right| \approx 0.05 \mathrm{GeV}^{2}$ the differential cross section is about $25 \%$ smaller than the calculation without Fermi motion and Pauli blocking (dotted curve). The reason for this reduction is that in the case that the bound nucleon moves towards the incoming photon the outgoing nucleon might be Pauli blocked for small momentum transfers. Note that this Pauli blocking just means that the whole nucleus absorbs the transferred momentum, i.e. this event contributes to the coherent production cross section which we do not consider here.

In Fig. 4 we show the transparency ratio $T_{A}$ for exclusive $\rho^{0}$ production as a function of the coherence length $l_{\rho}=q_{\rho}^{-1}$. The solid line is the result that one gets if one uses Eq. (2.12) and accounts for two-body correlations by making the substitution [18] 


$$
n\left(\vec{b}, z_{i}\right) \rightarrow n\left(\vec{b}, z_{i}\right)\left(1-j_{0}\left(q_{c}\left|z_{i}-z\right|\right)\right)
$$

in (2.12) with $q_{c}=0.78 \mathrm{GeV}$. This is necessary to avoid unphysical contributions from processes where $z_{i} \approx z$ which would contribute for small values of the coherence length $l_{\rho}$. In Ref. [18] we showed that this Bessel function parameterization yields a good description of shadowing in photoabsorption. In Eq. (2.12) we use for the total $\rho^{0} N$ cross section $\sigma_{\rho^{0}}=25 \mathrm{mb}$ and for the elastic part $\sigma_{e l}=3 \mathrm{mb}$. These two values correspond to the $\rho N$ cross sections used within the transport model for the involved $\rho^{0}$ momenta.

The result of the transport model is represented by the open squares. For each HERMES data point [3] we have made a separate calculation with the corresponding $\nu$ and $Q^{2}$. In the case of ${ }^{14} \mathrm{~N}$ the Glauber and the transport calculation are in perfect agreement with each other and the experimental data. This demonstrates that, as we have discussed in Ref. [4], Glauber theory can be used for the FSI if the right kinematic constraints are applied. For comparison we also show the result of Huefner et al. [8] (dashed curve) as well as the result that one gets when using the approximate expression by Yennie [11] including two-body correlations (dotted curve). The somewhat larger transparency ratio of Huefner et al. arises from the different density distribution that the authors of Ref. [8] use which reduces the effect of FSI. The difference of the Yennie result (dotted curve) and our calculation arises mainly from the different cross section in the FSI (second replacement in (2.13)). The different phase factor leads to the change in the transparency ratio at small coherence lengths, since $q_{V}=l_{V}^{-1}$.

After applying all of the above cuts, nearly all of the detected $\rho^{0}$ stem from diffractive $\rho^{0}$ production for which the formation time is zero. The ${ }^{14} \mathrm{~N}$ data seems to support the assumption that the time needed to put the preformed $\rho^{0}$ fluctuation on its mass shell and let the wave function evolve to that of a physical $\rho^{0}$ is small for the considered values of $Q^{2}$. Furthermore, the photon energy is too low to yield a large enough $\gamma$ factor to make the formation length exceed the internucleon distance and make color transparency visible. This conclusion is at variance with that reached in Ref. [6].

We now turn to ${ }^{84} \mathrm{Kr}$ where we expect a stronger effect of the FSI. Unfortunately there is yet no data available to compare with. As can be seen from Fig. 4 the transport calculation for ${ }^{84} \mathrm{Kr}$ gives a slightly smaller transparency ratio than the Glauber calculation, especially at low values of the coherence length, i.e. small momenta of the produced $\rho^{0}$. There are two reasons for this: About $10 \%$ of the difference arises from the fact that within the transport model the $\rho^{0}$ is allowed to decay into two pions. The probability that at least one of the pions interacts on its way out of the nucleus is about twice as large as that of the $\rho^{0}$. The other reason is that in the Glauber calculation (2.12) only the inelastic part of the $\rho^{0} N$ cross section enters whereas the transport calculation contains the elastic part as well. Thus all elastic scattering events out of the experimentally imposed $t$-window are neglected in the Glauber description. It is because of this $t$-window that also elastic $\rho^{0} N$ scattering reduces the transport transparency ratio shown in Fig. 4. Both effects are more enhanced at lower energies and become negligible for the much smaller ${ }^{14} \mathrm{~N}$ nucleus. 


\section{SUMMARY AND OUTLOOK}

We have developed a method to account for coherence length effects in incoherent electroproduction off nuclei which allows us to distinguish between the initial state interactions of the photon (shadowing) and the FSI of the reaction products. We have shown that our result is equivalent to the exact result of Glauber theory if one treats the FSI as a purely absorptive one. We have then performed a coupled channel treatment of the FSI within a semi-classical transport model and calculated the transparency ratio for exclusive incoherent $\rho^{0}$ photoproduction off ${ }^{14} \mathrm{~N}$ and ${ }^{84} \mathrm{Kr}$. The result for ${ }^{14} \mathrm{~N}$ is in agreement with experimental data and with the Glauber prediction. The latter shows that in the case of ${ }^{14} \mathrm{~N}$ Glauber theory is applicable after the kinematic cuts of the HERMES experiment are applied. Since we do not use a formation time for diffractively produced vector mesons we deduce that one cannot see an onset of color transparency in the nitrogen data. For the ${ }^{84} \mathrm{Kr}$ target no experimental data is available to compare with. However, we find deviations from the simple Glauber model because of the finite life time of the $\rho^{0}$ and elastic scattering out of the kinematically allowed $|t|$-region. These effects should be taken into account when evaluating the ${ }^{84} \mathrm{Kr}$ data in search of color transparency. As discussed by Kopeliovich et al. [6] one might see an onset of color transparency when investigating the transparency ratio as a function of $Q^{2}$ for fixed coherence length [3]. Including a formation time for exclusive $V$ production is not straightforward within our model since a change of the $V N$ cross section during the vector meson formation will influence both the initial as well as the final state interactions. This is planned for future work, when hopefully also the ${ }^{84} \mathrm{Kr}$ data has become available.

\section{ACKNOWLEDGMENTS}

The authors want to thank A. Borissov and W. Cassing for useful discussions. This work was supported by DFG.

\section{APPENDIX: A}

In the following we show the equality of Eqs. (2.12) and (2.14). Since the real part of the $V N$ scattering amplitude was neglected in the derivation of (2.14) we also set $\alpha_{V}=0$ in Equation (2.12) and end up with:

$$
\begin{aligned}
\sigma_{\gamma A \rightarrow V A^{*}}= & \sigma_{\gamma N \rightarrow V N} \int d^{2} b \int_{-\infty}^{\infty} d z n(\vec{b}, z) e^{-\sigma_{V}^{i n e l} \int_{z}^{\infty} d z^{\prime} n\left(\vec{b}, z^{\prime}\right)} \\
& \times\left|1-\int_{-\infty}^{z} d z_{i} n\left(\vec{b}, z_{i}\right) \frac{\sigma_{V}}{2} e^{i q_{V}\left(z_{i}-z\right)} \exp \left[-\frac{1}{2} \sigma_{V} \int_{z_{i}}^{z} d z_{k} n\left(\vec{b}, z_{k}\right)\right]\right|^{2} \\
= & \sigma_{\gamma N \rightarrow V N} \int d^{2} b \int_{-\infty}^{\infty} d z n(\vec{b}, z) e^{-\sigma_{V}^{i n e l} \int_{z}^{\infty} d z^{\prime} n\left(\vec{b}, z^{\prime}\right)}
\end{aligned}
$$




$$
\begin{aligned}
& \times\left\{1-\sigma_{V} \int_{-\infty}^{z} d z_{i} n\left(\vec{b}, z_{i}\right) \cos \left[q_{V}\left(z_{i}-z\right)\right] \exp \left[-\frac{1}{2} \sigma_{V} \int_{z_{i}}^{z} d z_{k} n\left(\vec{b}, z_{k}\right)\right]\right. \\
& \left.+\frac{\sigma_{V}^{2}}{4}\left|\int_{-\infty}^{z} d z_{i} n\left(\vec{b}, z_{i}\right) e^{i q_{V} z_{i}} \exp \left[-\frac{1}{2} \sigma_{V} \int_{z_{i}}^{z} d z_{k} n\left(\vec{b}, z_{k}\right)\right]\right|^{2}\right\} \\
& =\sigma_{\gamma N \rightarrow V N} \int d^{2} b\left\{\int_{-\infty}^{\infty} d z n(\vec{b}, z) e^{-\sigma_{V}^{i n e l} T_{z}(\vec{b})}\right. \\
& -\sigma_{V} \int_{-\infty}^{\infty} d z_{1} n\left(\vec{b}, z_{1}\right) \int_{z_{1}}^{\infty} d z_{2} n\left(\vec{b}, z_{2}\right) \cos \left[q_{V}\left(z_{1}-z_{2}\right)\right] \\
& \times \exp \left[-\sigma_{V}^{\text {inel }} \int_{z_{2}}^{\infty} d z^{\prime} n\left(\vec{b}, z^{\prime}\right)-\frac{1}{2} \sigma_{V} \int_{z_{1}}^{z_{2}} d z^{\prime} n\left(\vec{b}, z^{\prime}\right)\right] \\
& +\frac{\sigma_{V}^{2}}{4} \int_{-\infty}^{\infty} d z n(\vec{b}, z) \\
& \left.\times\left|\int_{-\infty}^{z} d z_{i} n\left(\vec{b}, z_{i}\right) e^{i q_{V} z_{i}} \exp \left[-\frac{1}{2} \sigma_{V} \int_{z_{i}}^{z} d z^{\prime} n\left(\vec{b}, z^{\prime}\right)-\frac{1}{2} \sigma_{V}^{\text {inel }} \int_{z}^{\infty} d z^{\prime} n\left(\vec{b}, z^{\prime}\right)\right]\right|^{2}\right\} .
\end{aligned}
$$

In the second step we have renamed the integration variables and rewritten the integral limits. The first term of (A1) already equals that the first term in (2.14). The exponent in the second term of (A1) yields

$$
-\sigma_{V}^{\text {inel }} T_{z_{2}}(\vec{b})-\frac{1}{2} \sigma_{V}\left(T_{z_{1}}(\vec{b})-T_{z_{2}}(\vec{b})\right)=-\frac{1}{2}\left(\sigma_{V}^{i n e l}-\sigma_{V}^{e l}\right) T_{z_{2}}(\vec{b})-\frac{1}{2} \sigma_{V} T_{z_{1}}(\vec{b})
$$

and the exponent of the third term in (A1) is

$$
-\frac{1}{2}\left(\sigma_{V} T_{z_{i}}(\vec{b})-T_{z}(\vec{b})\right)-\frac{1}{2} \sigma_{V}^{i n e l} T_{z}(\vec{b})=-\frac{1}{2} \sigma_{V} T_{z_{i}}(\vec{b})+\frac{1}{2} \sigma_{V}^{e l} T_{z}(\vec{b}) .
$$

We now further manipulate the last term of (A1):

$$
\begin{aligned}
& \frac{\sigma_{V}^{2}}{4} \int_{-\infty}^{\infty} d z n(\vec{b}, z) e^{\sigma_{V}^{e l} T_{z}(\vec{b})}\left|\int_{-\infty}^{z} d z_{i} n\left(\vec{b}, z_{i}\right) e^{i q_{V} z_{i}} e^{-\frac{1}{2} \sigma_{V} T_{z_{i}}(\vec{b})}\right|^{2} \\
& \quad=\frac{\sigma_{V}^{2}}{4} \int_{-\infty}^{\infty} d z \int_{-\infty}^{z} d z_{1} \int_{-\infty}^{z} d z_{2} n(\vec{b}, z) n\left(\vec{b}, z_{1}\right) n\left(\vec{b}, z_{2}\right) e^{\sigma_{V}^{e l} T_{z}(\vec{b})} \cos \left[q_{V}\left(z_{1}-z_{2}\right)\right] e^{-\frac{1}{2} \sigma_{V}\left(T_{z_{1}}(\vec{b})+T_{z_{2}}(\vec{b})\right)} .
\end{aligned}
$$

Rewriting the integral in the following form 


$$
\int_{-\infty}^{\infty} d z \int_{-\infty}^{z} d z_{1} \int_{-\infty}^{z} d z_{2}=\int_{-\infty}^{\infty} d z_{2} \int_{-\infty}^{z_{2}} d z_{1} \int_{z_{2}}^{\infty} d z+\int_{-\infty}^{\infty} d z_{1} \int_{-\infty}^{z_{1}} d z_{2} \int_{z_{1}}^{\infty} d z
$$

and using the symmetry of the integrand with respect to the variables $z_{1}$ and $z_{2}$ we can rewrite (A4) as

$$
\begin{aligned}
\frac{\sigma_{V}^{2}}{2} \int_{-\infty}^{\infty} d z_{2} n\left(\vec{b}, z_{2}\right) \int_{-\infty}^{z_{2}} d z_{1} n\left(\vec{b}, z_{1}\right) \cos \left[q_{V}\left(z_{1}-z_{2}\right)\right] e^{-\frac{1}{2} \sigma_{V}\left(T_{z_{1}}(\vec{b})+T_{z_{2}}(\vec{b})\right)} \int_{z_{2}}^{\infty} d z n(\vec{b}, z) e^{\sigma_{V}^{e l} T_{z}(\vec{b})} \\
=-\frac{1}{2} \frac{\sigma_{V}^{2}}{\sigma_{V}^{e l}} \int_{-\infty}^{\infty} d z_{2} n\left(\vec{b}, z_{1}\right) \int_{-\infty}^{z_{2}} d z_{1} n\left(\vec{b}, z_{2}\right) \cos \left[q_{V}\left(z_{1}-z_{2}\right)\right] e^{-\frac{1}{2} \sigma_{V}\left(T_{z_{1}}(\vec{b})+T_{z_{2}}(\vec{b})\right)}\left(1-e^{\sigma_{V}^{e l} T_{z_{2}}}\right) \\
=-\frac{1}{2} \frac{\sigma_{V}^{2}}{\sigma_{V}^{e l}} \int_{-\infty}^{\infty} d z_{1} n\left(\vec{b}, z_{1}\right) \int_{z_{1}}^{\infty} d z_{2} n\left(\vec{b}, z_{2}\right) \cos \left[q_{V}\left(z_{1}-z_{2}\right)\right] e^{-\frac{1}{2} \sigma_{V}\left(T_{z_{1}}(\vec{b})+T_{z_{2}}(\vec{b})\right)} \\
\quad+\frac{1}{2} \frac{\sigma_{V}^{2}}{\sigma_{V}^{e l}} \int_{-\infty}^{\infty} d z_{1} n\left(\vec{b}, z_{1}\right) \int_{z_{1}}^{\infty} d z_{2} n\left(\vec{b}, z_{2}\right) \cos \left[q_{V}\left(z_{1}-z_{2}\right)\right] e^{\left.-\frac{1}{2}\left(\sigma_{V}^{i n e l}-\sigma_{V}^{e l}\right) T_{z_{2}}(\vec{b})-\frac{1}{2} \sigma_{V} T_{z_{1}}(\vec{b})\right)}
\end{aligned}
$$

In the first step we have performed the integral over $z$. In the last step we have again rewritten the integral over $z_{1}$ and $z_{2}$ to be able to combine the second term of (A6) with the second term of (A1) to get the second term in (2.14). Now we only have to show the equality of the last term in (2.14) and the first one in (A6). Therefore we rewrite

$$
\begin{aligned}
& -\frac{1}{4} \frac{\sigma_{V}^{2}}{\sigma_{V}^{e l}}\left|\int_{-\infty}^{\infty} d z n(\vec{b}, z) e^{i q_{V} z} e^{-\frac{1}{2} \sigma_{V} T_{z}(\vec{b})}\right|^{2} \\
& \quad=-\frac{1}{4} \frac{\sigma_{V}^{2}}{\sigma_{V}^{e l}} \int_{-\infty}^{\infty} d z_{1} \int_{-\infty}^{\infty} d z_{2} n\left(\vec{b}, z_{1}\right) n\left(\vec{b}, z_{2}\right) \cos \left[q_{V}\left(z_{1}-z_{2}\right)\right] e^{-\frac{1}{2} \sigma_{V}\left(T_{z_{1}}(\vec{b})+T_{z_{2}}(\vec{b})\right)}
\end{aligned}
$$

and reformulate the integral as

$$
\begin{aligned}
\int_{-\infty}^{\infty} d z_{1} \int_{-\infty}^{\infty} d z_{2} & =\int_{-\infty}^{\infty} d z_{1} \int_{z_{1}}^{\infty} d z_{2}+\int_{-\infty}^{\infty} d z_{2} \int_{z_{2}}^{\infty} d z_{1} \\
& =2 \int_{-\infty}^{\infty} d z_{1} \int_{z_{1}}^{\infty} d z_{2}
\end{aligned}
$$

where the second equality again follows from the symmetry of the integrand with respect to the exchange of $z_{1}$ and $z_{2}$. The two expressions (2.12) and (2.14) are therefore equivalent. 


\section{REFERENCES}

[1] A. H. Mueller, in Proceedings of the 17th Recontre de Moriond, Moriond, 1982, edited by J. Tran Tanh Van (Editions Frontieres, Gif-sur-Yvette Cedex, France, 1982), p.13.; S. J. Brodsky, in Proceedings of the 13th Symposium of Multiparticle Dynamics, edited by W. Kittel, W. Metzger, and A. Stergiou (World Scientific, Singapore, 1982).

[2] K. Ackerstaff et al., HERMES Collaboration, Phys. Rev. Lett. 82, 3025 (1999).

[3] A. Airapetian et al., HERMES Collaboration, Phys. Rev. Lett. 90, 052501 (2003).

[4] T. Falter and U. Mosel, Phys. Rev. C 66, 024608 (2002).

[5] R. J. Glauber, in Lectures in Theoretical Physics, edited by W.E. Brittin and L.G. Dunham (Wiley Interscience, New York, 1959), Vol. I, p. 315.

[6] B. Z. Kopeliovich, J. Nemchik, A. Schaefer, and A. V. Tarasov, Phys. Rev. C 65, 035201 (2001).

[7] P. Jain, B. Pire, and J. P. Ralston, Phys. Rep. 271, 67 (1996).

[8] J. Hüfner, B. Kopeliovich, and J. Nemchik, Phys Lett. B 383, 362 (1996).

[9] C. Friberg and T. Sjostrand, JHEP 0009, 010 (2000).

[10] T. Sjostrand, P. Eden, C. Friberg, L. Lonnblad, G. Miu, S. Mrenna, and E. Norrbin, Comput. Phys. Commun.135, 238 (2001); T. Sjostrand, L. Lonnblad, and S. Mrenna, [hep-ph/0108264].

[11] D. R. Yennie, in Hadronic Interactions of Electrons and Photons, edited by J. Cummings and H. Osborn (Academic, New York/London, 1971), p. 321; T. H. Bauer, F. Pipkin, R. Spital, and D. R. Yennie, Rev. Mod. Phys. 50, 261 (1978).

[12] M. Effenberger, E. L. Bratkovskaya, and U. Mosel, Phys. Rev. C 60, 044614 (1999).

[13] A. Airapetian et al., HERMES Collaboration, Eur. Phys. J. C 17, 389 (2000).

[14] M. R. Adams et al., E665 Collaboration, Z. Phys. C 74, 237 (1997).

[15] W. D. Shambroom et al., CHIO Collaboration, Phys. Rev. D 26, 1 (1982).

[16] M. R. Adams et al., E665 Collaboration, Phys. Rev. Lett. 74, 1525 (1995).

[17] G. McClellan et al., Phys. Rev. Lett. 23, 554 (1969).

[18] T. Falter, S. Leupold, and U. Mosel, Phys. Rev. C 62, 031602(R) (2000). 


\section{FIGURES}

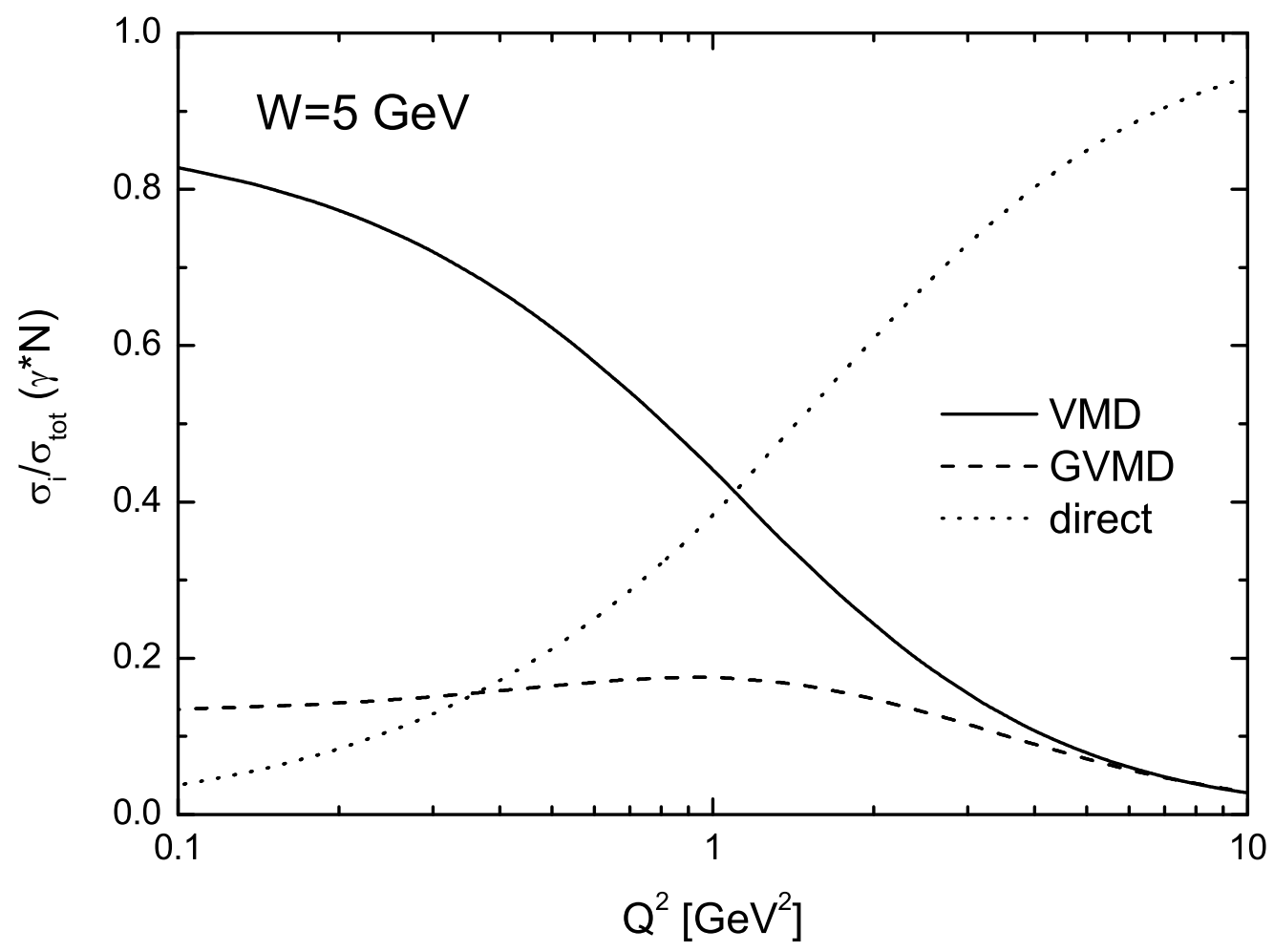

FIG. 1. PYTHIA result for the fraction of the total $\gamma^{*} N$ cross section that is contributed by the VMD (solid line), GVMD (dashed) and direct (dotted) part of the photon as a function of $Q^{2}$ for fixed invariant mass $W=5 \mathrm{GeV}$. Note that in contrast to the authors of Ref. [9] we call every pointlike interaction of the photon a direct process. 


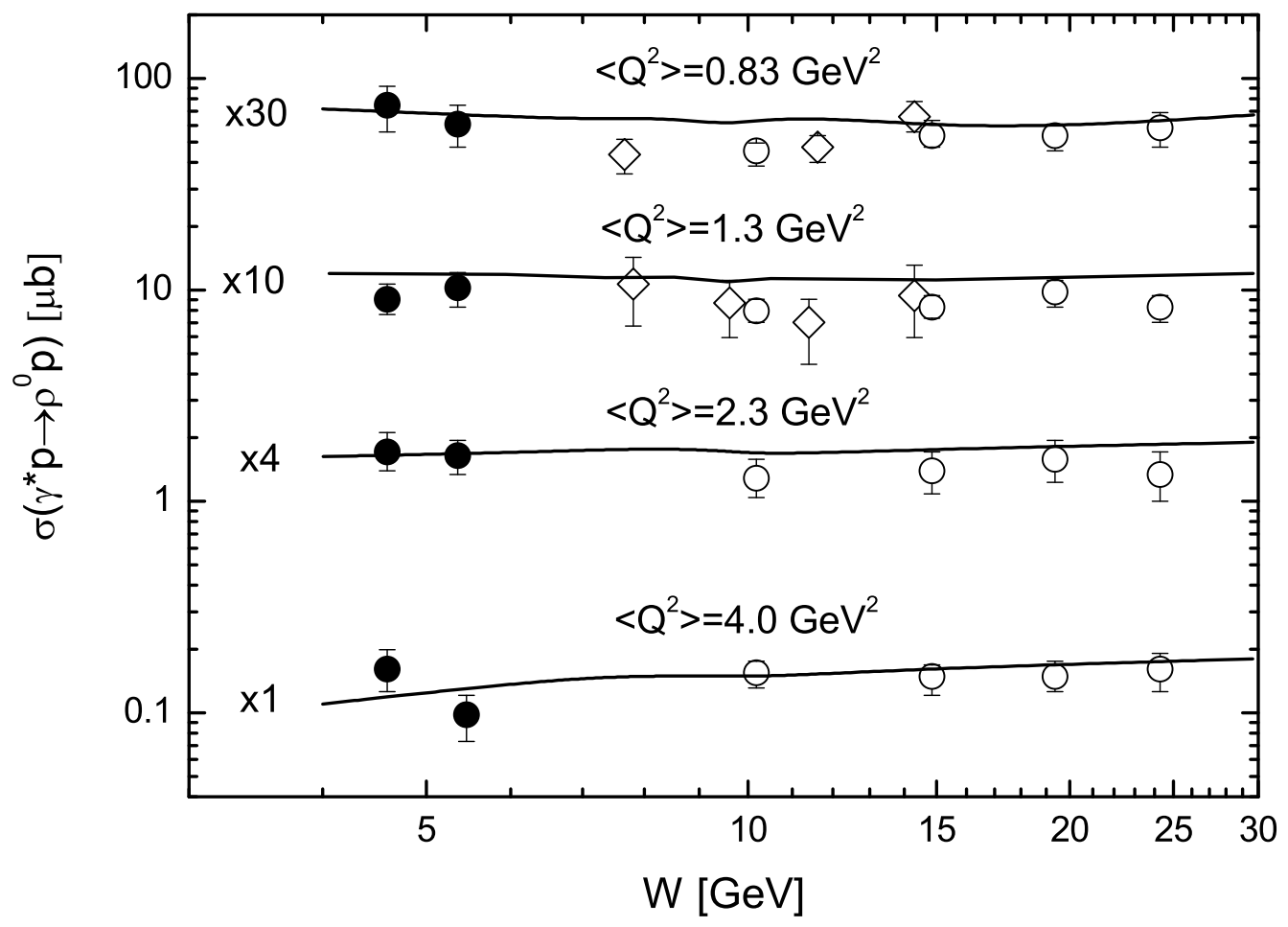

FIG. 2. The virtual-photoproduction cross section for $\rho^{0}$ production plotted versus the invariant mass $W$ at average $Q^{2}$ values of $0.83,1.3,2.3$ and $4.0 \mathrm{GeV}^{2}$. The data has been taken from the HERMES [13] (filled circles), E665 [14] (open circles) and CHIO collaboration [15] (open diamonds). 


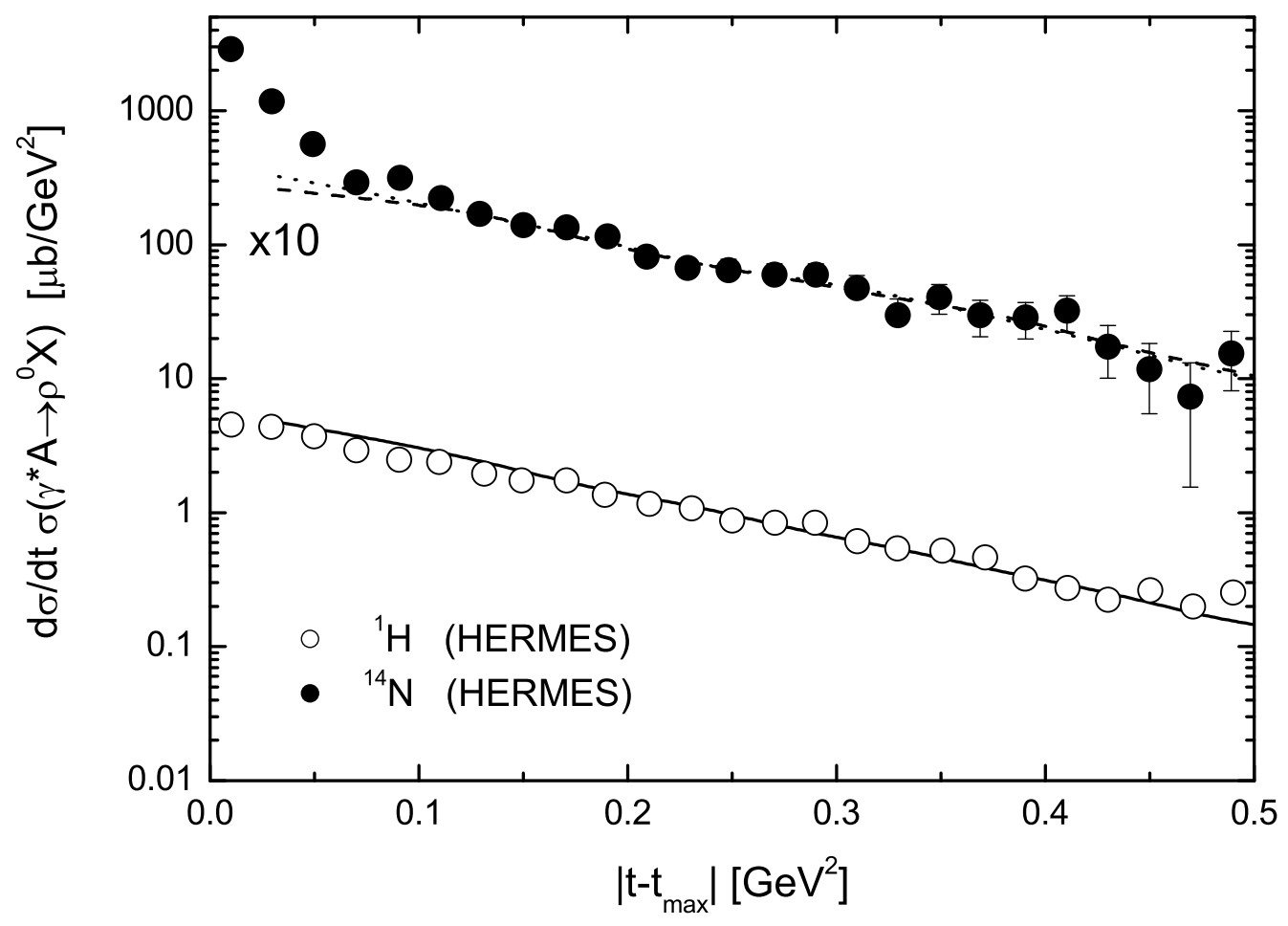

FIG. 3. The exclusive incoherent differential $\rho^{0}$ production cross section for ${ }^{1} H$ (solid line) and ${ }^{14} \mathrm{~N}$ (dashed line) in comparison with experimental data from the HERMES collaboration [2]. The calculation has been performed for $\nu=13 \mathrm{GeV}$ and $Q^{2}=1.7 \mathrm{GeV}^{2}$. The dotted curve represents a calculation for ${ }^{14} \mathrm{~N}$ without Pauli blocking and Fermi motion. For details on the exclusivity measure see text. 


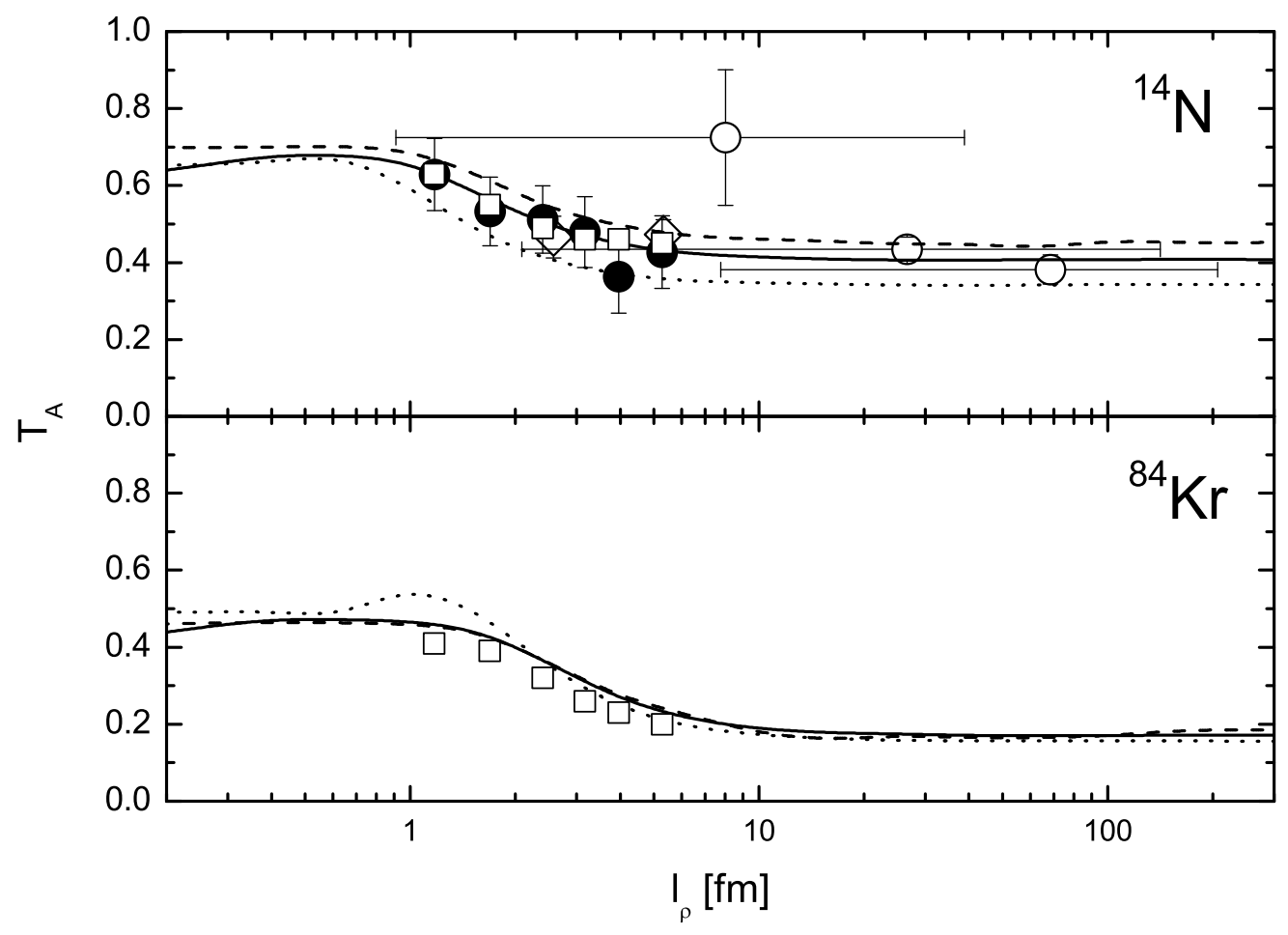

FIG. 4. Nuclear transparency ratio $T_{A}$ for $\rho^{0}$ electroproduction plotted versus the coherence length of the $\rho^{0}$ component of the photon. The data is taken from [3] (filled circles), [16] (open circles) and [17] (open diamonds). The solid line represents the Glauber result when using (2.12) including nucleon-nucleon correlations, the dotted line shows the effect of the substitutions (2.13). The dashed curve represents the Glauber result using (2.14) with the density distribution of Ref. [8]. For each transparency ratio calculated within our transport model (open squares) we used the average value of $Q^{2}$ and $\nu$ of the corresponding HERMES data point. 